Check for updates

Cite this: RSC Adv., 2018, 8, 21728

Received 18th April 2018

Accepted 25th May 2018

DOI: 10.1039/c8ra03331e

rsc.li/rsc-advances

\title{
Atomic oxygen effects on silvered polyimide films and their surface modification by poly(siloxane amic acid) ammonium salts
}

\author{
Wang Chunbo, ${ }^{\text {ab }}$ Li Liaoliao, ${ }^{c}$ Jiang Haifu, ${ }^{d}$ Tian Dongbo, ${ }^{d}$ Qin Wei, ${ }^{d}$ Liu Changwei, ${ }^{c}$ \\ Zhao Xiaogang, ${ }^{\text {ab }}$ Chen Chunhai ${ }^{\text {ab }}$ and Wang Daming (D) *ab
}

\begin{abstract}
The tolerance of silvered polyimide films synthesized by an in situ self-metalization method against atomic oxygen (AO) was evaluated. The results showed that the mass loss of R-Ag/PI was markedly increased as the $A O$ fluence increased; $\mathrm{Ag} / \mathrm{PI}$ showed an identical trend. SEM data showed that the silver particles on the surfaces of R-Ag/PI and Ag/PI disappeared. The surfaces achieved a "carpet condition" that was more obvious as the AO fluence increased. Poly(siloxane amic acid) ammonium salt was synthesized and made via imidization to produce a flexible organic coating that was characterized by ATR-FTIR, ${ }^{1} \mathrm{HNMR}$, TGA, and XPS. This could be used to improve the tolerance of silvered polyimide films against AO. The $A O$ resistance and the impacts on mass loss, surface morphology, and surface compositions were also evaluated after surface modification by poly(siloxane amic acid) ammonium salts. $20 \mathrm{wt} \% \mathrm{Foc} / \mathrm{Ag} / \mathrm{PI}$ had a lower mass loss and smoother surface than the others due to the formation of a compact surface$\mathrm{SiO}_{2}$-type layer. This flexible organic coating can be produced via an environmentally-friendly method, and it maintains the inherent thermal stability of the polyimide which cannot be achieved by other anti$\mathrm{AO}$ coatings.
\end{abstract}

\section{Introduction}

Polyimide (PI) is an important class of polymer with superior thermal stability, outstanding electrical properties, excellent mechanical properties, inertness to solvents and good adhesion properties. It has been applied in many fields, such as aerospace, functional membranes, microelectronics, and advanced composites. ${ }^{1-5}$ Silvered polyimide films are functional polymers that have been an active area of interest for an in situ selfmetalization method. They have potential applications in highly reflective thin film reflectors and concentrators in space environments for solar thermal propulsion and large-scale radio frequency antennas..$^{6-9}$

Numerous spacecraft, including the International Space Station (ISS) and Hubble Space Telescope (HST), are running in low-earth orbits (LEOs). In LEO, factors such as atomic oxygen (AO), ultraviolet (UV), ionizing radiation, thermal cycles, and orbital debris can degrade the polymers. ${ }^{10-13} \mathrm{AO}$ is one of the

${ }^{a}$ Key Laboratory of High Performance Plastics (Jilin University), Ministry of Education, College of Chemistry, Jilin University, Changchun, 130012, P. R. China. E-mail: wangdaming@jlu.edu.cn

${ }^{b}$ National \& Local Joint Engineering Laboratory for Synthesis Technology of High Performance Polymer, College of Chemistry, Jilin University, Changchun, 130012, P. R. China

'Institute of Petroleum Chemistry, Heilongjiang Academy of Sciences, Harbin 150040, China

${ }^{d}$ Beijing Institute of Satellite Environment Engineering, Beijing 100094, China harshest environmental factors, and it erodes exposed materials. ${ }^{14-16}$ Hence, before silvered polyimide films can be used in LEO, evaluation of the tolerance of silvered polyimide films against $\mathrm{AO}$ is necessary. ${ }^{17,18}$ Silver is prone to erosion by $\mathrm{AO}$, and the primary mechanism is oxidation by atomic oxygen to form porous silver oxide.

Coatings are a common approach to improving the AO resistance of materials. ${ }^{16}$ Flexible organic coatings can avoid the defects of hard inorganic coatings that affect AO resistance. These very fragile defects are generated during production. Flexible organic coatings can offer excellent AO erosion resistance and properties that are particularly important for longterm space applications. ${ }^{19,20}$

Here, we evaluated the tolerance of silvered polyimide films synthesized by an in situ self-metalization method against AO. Surface modification by poly(siloxane amic acid) ammonium salts (PAAS) made by imidization produced flexible organic coatings. We also evaluated the AO resistance after surface modification by poly(siloxane amic acid) ammonium salts at different solid contents. The metrics include mass loss, surface morphology and surface composition.

\section{Experimental}

\subsection{Materials}

Commercial Kapton polyimide films (Dupont, $50 \mu \mathrm{m}$ ) were used. Potassium hydroxide $(\mathrm{KOH})$, silver nitrate $\left(\mathrm{AgNO}_{3}\right)$, ethanol, 


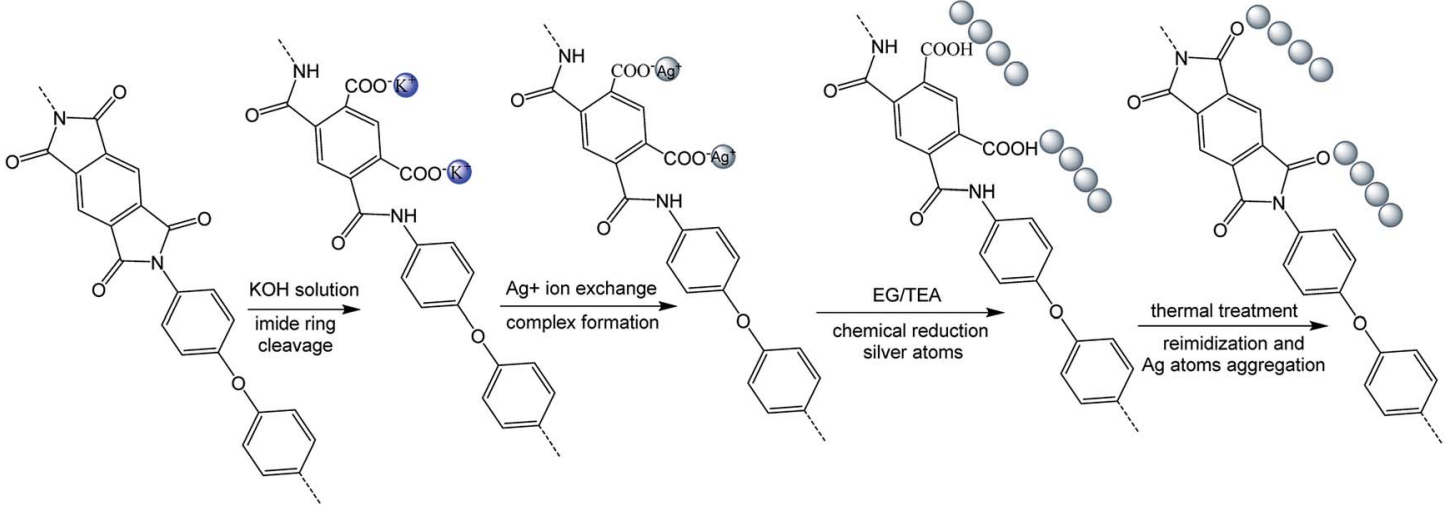

Scheme 1 Preparation of the self-metalized films.

triethylamine (TEA), and ethylene glycol (EG) were purchased from Acros and used without further purification. Bis( $\gamma$-aminopropyl $)$ polydimethylsiloxane $\left(M_{\mathrm{n}}=860 \mathrm{~g} \mathrm{~mol}^{-1}\right)$ was supplied by Gelest Inc., and was used as received. $3,3^{\prime}, 4,4^{\prime}$-Biphenyltetracarboxylic dianhydride was purchased from Sinopharm Chemical Reagent Beijing Co. Ltd and baked at $110{ }^{\circ} \mathrm{C}$ in a vacuum overnight prior to use.

\subsection{Characterization}

${ }^{1} \mathrm{H}$ nuclear magnetic resonance (NMR) spectra were measured on a BRUKER-300 spectrometer at $300 \mathrm{MHz}$. Thermogravimetric analysis (TGA) was tested with a TA 2050 at a heating rate of $10{ }^{\circ} \mathrm{C} \mathrm{min}^{-1}$ under air. The surface composition and surface chemical states were measured via X-ray photoelectron spectroscopy (XPS) by using an Escalab 250. The AO irradiation tests were performed using a microwave source $\mathrm{AO}$ ground simulation device, and a typical AO flux was estimated to be $2.5 \times 10^{14}$ atoms per $\left(\mathrm{cm}^{2} \mathrm{~s}\right)$. Samples were weighed using an electronic balance (AB135-S, METTLER TOLEDO) at room temperature immediately after the AO irradiation tests. The mass of the sample was taken as the average of three measurements. Mass loss was defined as the difference in value of the mass before and after exposure to AO. The AO-irradiated sample surfaces were observed using scanning electron microscopy (SEM, NOVA NANOSEM 450, England). Samples used for surface morphology observation were deposited on $c a .5 \mathrm{~nm}$ platinum (Pt) prior to measurement.

\subsection{Preparation of the self-metalized films ${ }^{8}$}

First, polyimide films (Kapton, $50 \mu \mathrm{m}$ ) were immersed into aqueous $\mathrm{KOH}$ solution ( $4 \mathrm{M}$ ) for 15 minutes at room temperature and then washed with lots of deionized water. Second, the silver ions were incorporated into the surface-modified films via aqueous $\mathrm{AgNO}_{3}$ solution $(1 \mathrm{M})$ for $1 \mathrm{~h}$ at room temperature. The film was then washed with deionized water. Third, the silver ions were reduced by immersion of the films into the solution $(\mathrm{EG}: \mathrm{TEA}=10: 1)$ for 5 minutes at $90^{\circ} \mathrm{C}$. The surface-modified films were obtained after reduction and named R-Ag/PI.

Finally, $\mathrm{R}-\mathrm{Ag} / \mathrm{PI}$ was made by reimidization via thermal treatment $\left(100{ }^{\circ} \mathrm{C} / 1 \mathrm{~h} ; 200{ }^{\circ} \mathrm{C} / 1 \mathrm{~h} ; 300{ }^{\circ} \mathrm{C} / 1 \mathrm{~h}\right)$ to obtain the surface-modified films after reimidization, and named Ag/PI (see Scheme 1).

\subsection{Preparation of poly(siloxane amic acid) ammonium salt (PAAS) ${ }^{21}$}

$1.47 \mathrm{~g}$ of $3,3^{\prime}, 4,4^{\prime}$-biphenyltetracarboxylic dianhydride was slowly added to the solution of $\operatorname{bis}(\gamma$-aminopropyl)polydimethylsiloxane ( $4.30 \mathrm{~g}, 5 \mathrm{mmol}$ ) in $41 \mathrm{ml}$ of ethanol. Then, the reaction mixture was stirred at $-10{ }^{\circ} \mathrm{C}$ for $10 \mathrm{~h}$ under $\mathrm{N}_{2}$ protection. Subsequently, $1.01 \mathrm{~g}$ of triethylamine was added slowly $(0.5-1 \mathrm{~h})$, and the reaction mixture was stirred for another $4 \mathrm{~h}$. After drying under vacuum at $40-50{ }^{\circ} \mathrm{C}$ overnight, poly(siloxane amic acid) ammonium salt (PAAS) was obtained (see Scheme 2).

\subsection{Surface modification of Ag/PI by PAAS}

PAAS was dissolved in ethylene glycol with a little triethylamine at solid contents of 10 and $20 \mathrm{wt} \%$, respectively. Then, the solutions were spun on the surface of R-Ag/PI. The flexible organic coatings were obtained after imidization $\left(100{ }^{\circ} \mathrm{C} / 1 \mathrm{~h}\right.$; $200{ }^{\circ} \mathrm{C} / 1 \mathrm{~h} ; 300{ }^{\circ} \mathrm{C} / 1 \mathrm{~h}$ ) and named $10 \mathrm{wt} \%$ Foc/Ag/PI and $20 \mathrm{wt} \%$ Foc/Ag/PI (see Scheme 3).

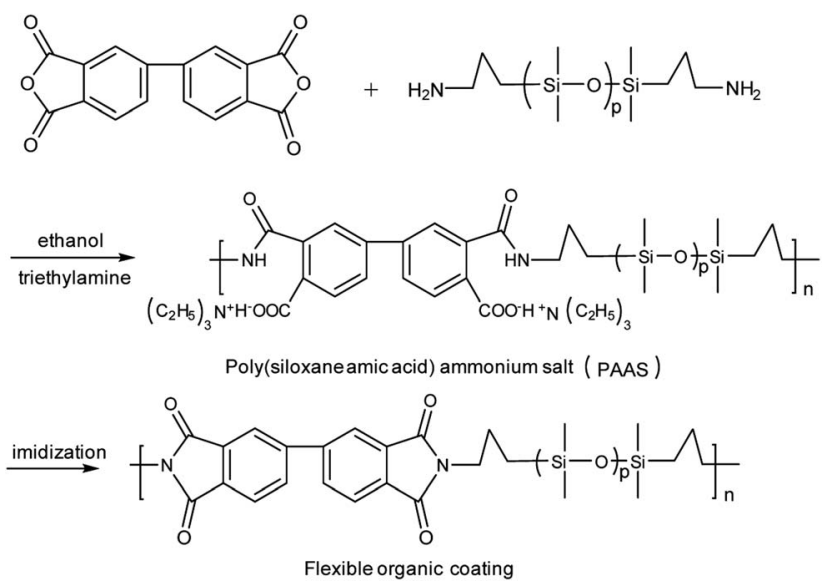

Scheme 2 Synthesis of PAAS and imidization to obtain flexible organic coatings. 


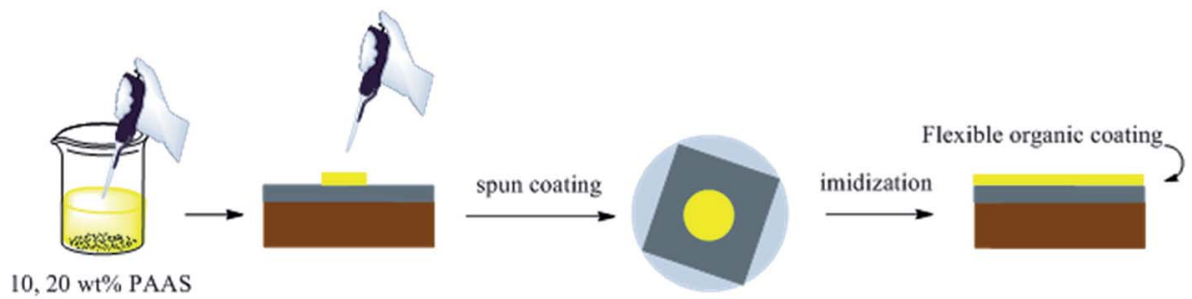

Scheme 3 Surface modification of the self-metalized films by PAAS.

\section{Results and discussion}

\subsection{Preparation of the samples}

Many groups have reported the primary mechanism of the selfmetalized reaction on the polyimide surface. Here, a selfmetalized film was prepared via the procedures of hydrolytic cleavage of the imide groups, incorporation of silver ions, reduction of silver ions and reimidization (see Scheme 1). The depth of the surface-modified film was $c a .5 \mu \mathrm{m}$, as measured by SEM via fracture morphology. The XPS results showed the bonding states of the silver on the surface of $\mathrm{R}-\mathrm{Ag} / \mathrm{PI}$ and $\mathrm{Ag} / \mathrm{PI}$. These were characteristic of metallic silver with peaks located at binding energies around $368.4 \mathrm{eV}$ (see Fig. 3). ${ }^{22}$

Compared to poly(amic acid), poly(amic acid) ammonium salt has hydrolytic stability and good solubility in some environmentally-friendly solvents. ${ }^{23}$ Meanwhile, polysiloxaneblock-polyimides possess good AO resistance, as reported in the literature. ${ }^{20}$ Therefore, poly(siloxane amic acid) ammonium salts were synthesized and spun on the surface of R-Ag/PI at solid contents of $10 \mathrm{wt} \%$ and $20 \mathrm{wt} \%$, and then imidized: 100 ${ }^{\circ} \mathrm{C} / 1 \mathrm{~h} ; 200{ }^{\circ} \mathrm{C} / 1 \mathrm{~h} ; 300{ }^{\circ} \mathrm{C} / 1 \mathrm{~h}$ (see Schemes 2 and 3) to obtain a flexible organic coating that can protect the silvered polyimide from AO attack. ATR-FTIR measurements were performed on $10 \mathrm{wt} \% \mathrm{Foc} / \mathrm{Ag} / \mathrm{PI}$ and $20 \mathrm{wt} \% \mathrm{Foc} / \mathrm{Ag} / \mathrm{PI}$, as shown in Fig. 1.

For the flexible organic coating, a relatively strong peak attributed to the $\mathrm{Si}-\mathrm{O}$ stretching of the siloxane appeared at 1016 and $1063 \mathrm{~cm}^{-1}$. The characteristic bands for the imide groups appeared at $1770,1710 \mathrm{~cm}^{-1}$, and $1380 \mathrm{~cm}^{-1}$. This

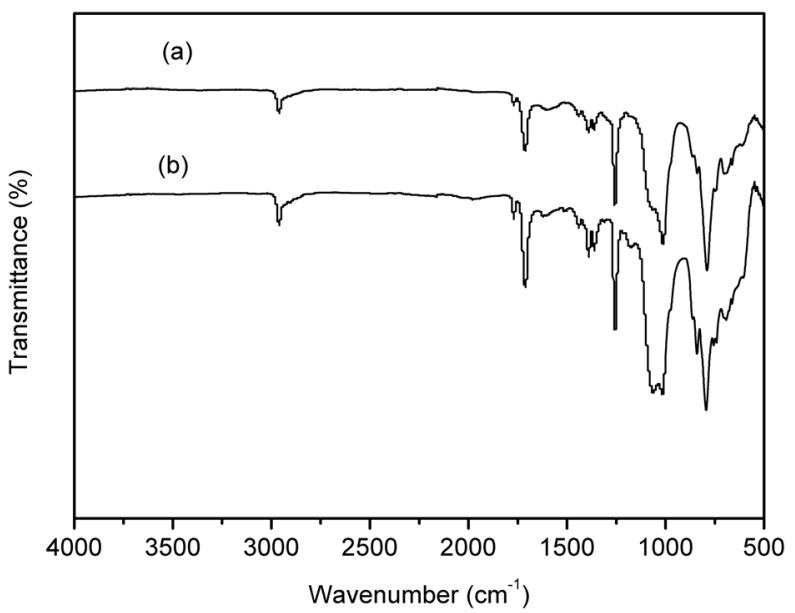

Fig. 1 ATR-FTIR spectra of (a) 10 wt\% Foc/Ag/PI and (b) 20 wt\% Foc/ $\mathrm{Ag} / \mathrm{PI}$. proved that PAAS formed polyimide. The absence of the characteristic peaks of PAAS at about $1660 \mathrm{~cm}^{-1}$ (amic acid $\mathrm{C}=\mathrm{O}$ stretching) and $1535 \mathrm{~cm}^{-1}$ (amide $\mathrm{C}-\mathrm{N}$ stretching) suggest complete imidization of PAAS. The flexible organic coating derived from $-\mathrm{CH}_{3}$ absorption had a peak at $2960 \mathrm{~cm}^{-1}$ and Si$\mathrm{CH}_{3}$ absorption was at 794 and $841 \mathrm{~cm}^{-1} \cdot{ }^{1} \mathrm{H}$ NMR was measured on a flexible organic coating, as shown in Fig. $2\left({ }^{1} \mathrm{H}\right.$ NMR $\left(400 \mathrm{MHz}, \mathrm{CDCl}_{3}\right) \delta 8.08(\mathrm{~s}, 2 \mathrm{H}), 7.95(\mathrm{~s}, 4 \mathrm{H}), 3.69(\mathrm{t}, J=$ $6.7 \mathrm{~Hz}, 4 \mathrm{H}), 1.71(\mathrm{~s}, 4 \mathrm{H}), 0.62-0.54(\mathrm{~m}, 4 \mathrm{H}), 0.22-0.00(\mathrm{~m}, 84 \mathrm{H}))$. The signals at $\delta 8.08$ and 7.95 are derived from benzene; signals of the propyl group appeared at $\delta 3.69,1.71$ and $0.62-0.54$; the relatively strong peak at $0.22-0.00$ was attributed to the methyl connecting with the silicon. The spectroscopic data obtained by ${ }^{1} \mathrm{H}$ NMR and ATR-FTIR agreed with the expected structures.

After surface modification by PAAS, the silver contents on the surfaces of $10 \mathrm{wt} \% \mathrm{Foc} / \mathrm{Ag} / \mathrm{PI}$ and $20 \mathrm{wt} \%$ Foc/Ag/PI decreased from $25.63 \%$ and $20.46 \%$ to $2.08 \%$ and $0 \%$, in Fig. 3 and 4 . This data showed that the silver cannot be completely covered via surface modification with a solid content of $10 \mathrm{wt} \%$ PAAS. However, surface modification with $20 \mathrm{wt} \%$ PAAS left no silver on the surface.

\subsection{Thermal stability of the samples}

TGA curves of the samples: (a) R-Ag/PI; (b) Ag/PI; (c) $10 \mathrm{wt} \%$ Foc/ $\mathrm{Ag} / \mathrm{PI}$; (d) $20 \mathrm{wt} \% \mathrm{Foc} / \mathrm{Ag} / \mathrm{PI}$ are listed in Fig. 5, and the decomposition temperature $\left(T_{\mathrm{d}}\right)$ at $10 \%$ weight loss in an air atmosphere was determined from the original TGA curves and

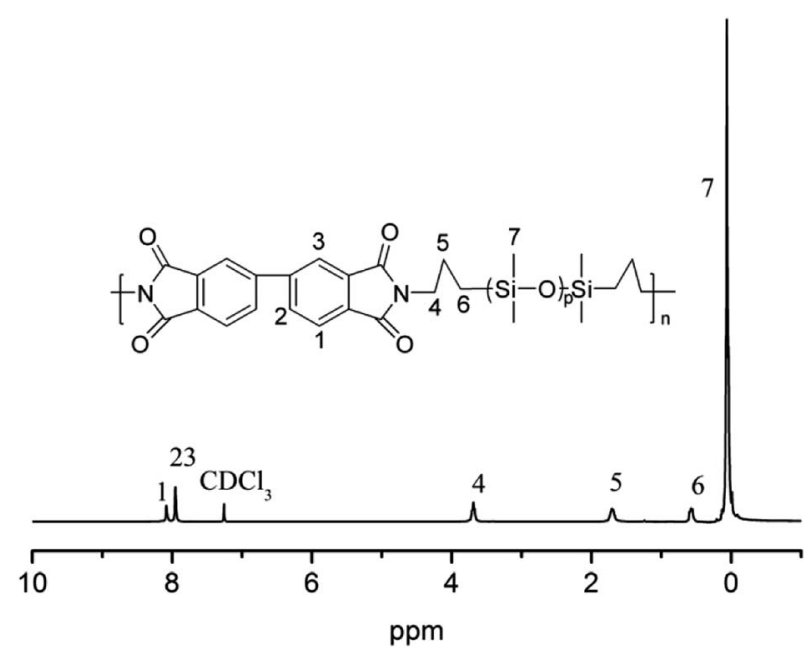

Fig. $2{ }^{1} \mathrm{H}$ NMR spectrum of the flexible organic coating. 


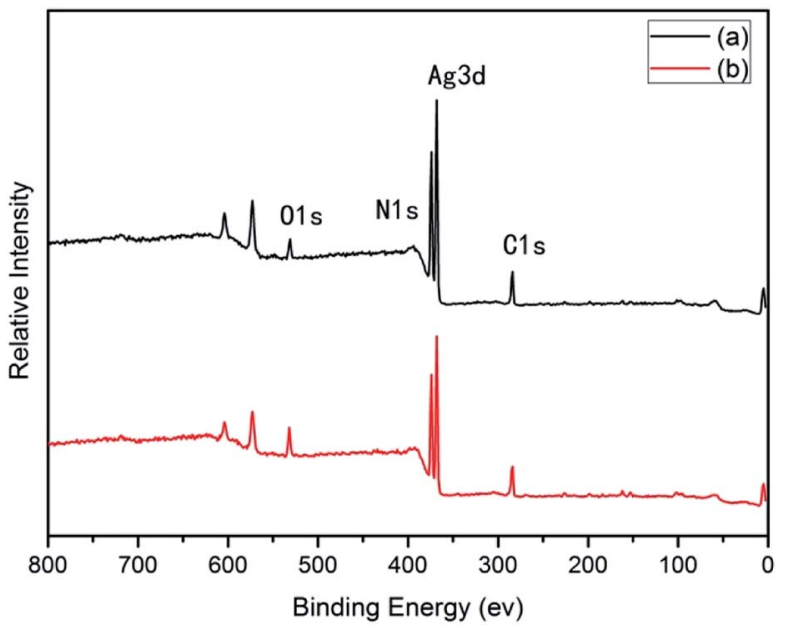

Fig. 3 XPS spectra of the samples: (a) R-Ag/PI; (b) $\mathrm{Ag} / \mathrm{PI}$.

is also given in Table 1 . The $10 \%$ weight loss temperatures $\left(T_{10 \%}\right)$ of $\mathrm{R}-\mathrm{Ag} / \mathrm{PI}$ and $\mathrm{Ag} / \mathrm{PI}$ in air were recorded at 409 and $518{ }^{\circ} \mathrm{C}$. Thermogravimetric analysis of the R-Ag/PI film shows some mass loss around $200-250{ }^{\circ} \mathrm{C}$ due to the existence of residual solvent and amic acids. After reimidization, the Ag/PI had no mass loss under $400{ }^{\circ} \mathrm{C}$. The $10 \%$ weight loss temperatures were markedly increased after reimidization $(\mathrm{R}-\mathrm{Ag} / \mathrm{PI}$ versus $\mathrm{Ag} / \mathrm{PI}$ ). Hence, it is necessary that the surface-modified films after reduction be reimidized to improve the thermal stability of the self-metalized films. The $10 \%$ weight loss temperatures $\left(T_{10 \%}\right)$ of $10 \mathrm{wt} \% \mathrm{Foc} / \mathrm{Ag} / \mathrm{PI}$ and $20 \mathrm{wt} \% \mathrm{Foc} / \mathrm{Ag} / \mathrm{PI}$ in air were recorded at 445 and $436{ }^{\circ} \mathrm{C}$, respectively. The $10 \%$ weight loss temperatures obviously decreased after surface modification by PAAS, but they also had outstanding thermal stability compared with other organic silicon resins. A slight mass loss at around $200-250{ }^{\circ} \mathrm{C}$ was seen with imidization for $10 \mathrm{wt} \% \mathrm{Foc} / \mathrm{Ag} / \mathrm{PI}$ and $20 \mathrm{wt} \% \mathrm{Foc} / \mathrm{Ag} / \mathrm{PI}$. This is because PAAS was spun on the surface of $\mathrm{R}-\mathrm{Ag} / \mathrm{PI}$, and the protective layer may hinder the volatilization of the solvent existing in the substrate.

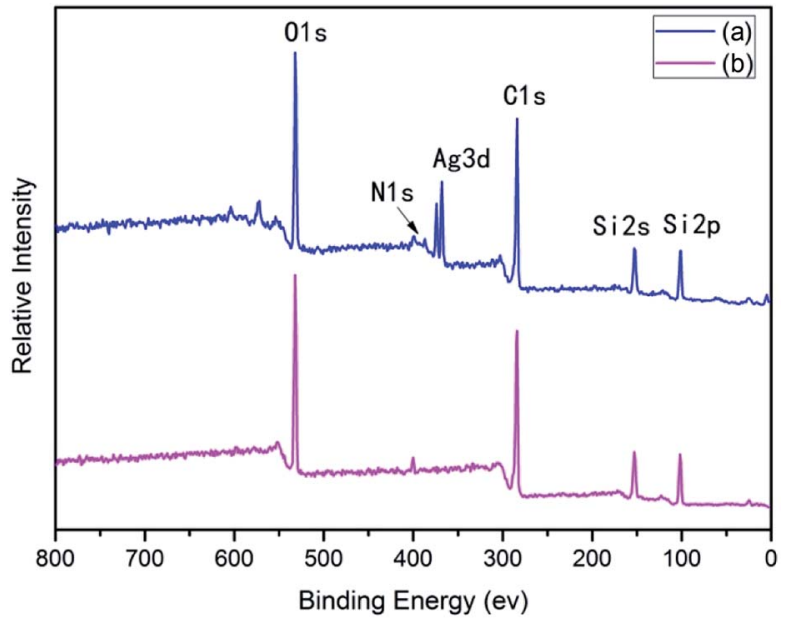

Fig. 4 XPS spectra of the samples: (a) 10 wt\% Foc/Ag/PI; (b) 20 wt\% $\mathrm{Foc} / \mathrm{Ag} / \mathrm{PI}$.

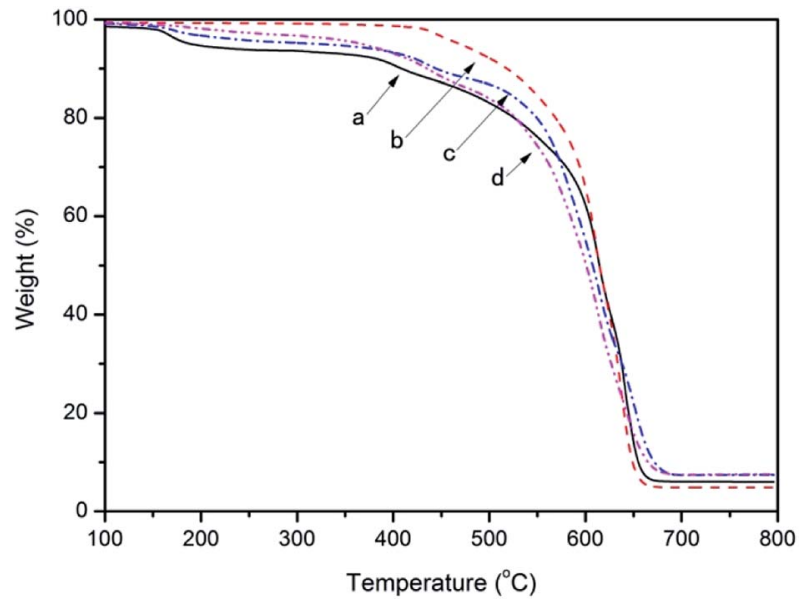

Fig. 5 TGA curves of the samples: (a) R-Ag/PI; (b) Ag/PI; (c) $10 w t \%$ Foc/Ag/PI; and (d) 20 wt\% Foc/Ag/PI.

\subsection{Surface morphology of the samples}

SEM data: (a) R-Ag/PI; (b) Ag/PI; (c) $10 \mathrm{wt} \% \mathrm{Foc} / \mathrm{Ag} / \mathrm{PI}$; (d) $20 \mathrm{wt} \% \mathrm{Foc} / \mathrm{Ag} / \mathrm{PI}$ are shown in Fig. 6. A number of silver particles on the $\mathrm{R}-\mathrm{Ag} / \mathrm{PI}$ and $\mathrm{Ag} / \mathrm{PI}$ surfaces were observed, and well-defined silver layers formed after re-imidization. The SEM pictures of $10 \mathrm{wt} \% \mathrm{Foc} / \mathrm{Ag} / \mathrm{PI}$ and $20 \mathrm{wt} \% \mathrm{Foc} / \mathrm{Ag} / \mathrm{PI}$ show that $10 \mathrm{wt} \%$ PAAS cannot fully cover the silver layers on the surface of self-metalized films, but silver particles cannot be seen after modification by $20 \mathrm{wt} \%$ PAAS. This phenomenon can also be illustrated with XPS data.

Table $1 T_{10 \%}$ of the samples derived from TGA curves

\begin{tabular}{ll}
\hline Samples & $T_{10 \%}\left({ }^{\circ} \mathrm{C}\right)$ \\
\hline $\mathrm{R}-\mathrm{Ag} / \mathrm{PI}$ & 409 \\
$\mathrm{Ag} / \mathrm{PI}$ & 518 \\
$10 \mathrm{wt} \% \mathrm{Foc} / \mathrm{Ag} / \mathrm{PI}$ & 445 \\
$20 \mathrm{wt} \%$ Foc/Ag/PI & 436
\end{tabular}

$20 \mathrm{wt} \% \mathrm{Foc} / \mathrm{Ag} / \mathrm{PI}$
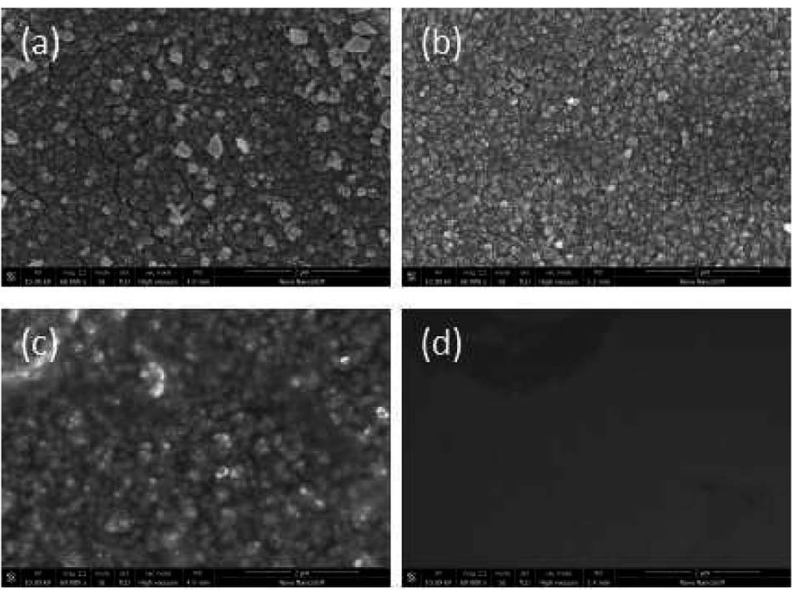

Fig. 6 SEM pictures of the samples: (a) R-Ag/PI; (b) Ag/PI; (c) 10 wt\% Foc/Ag/Pl; (d) 20 wt\% Foc/Ag/PI. 


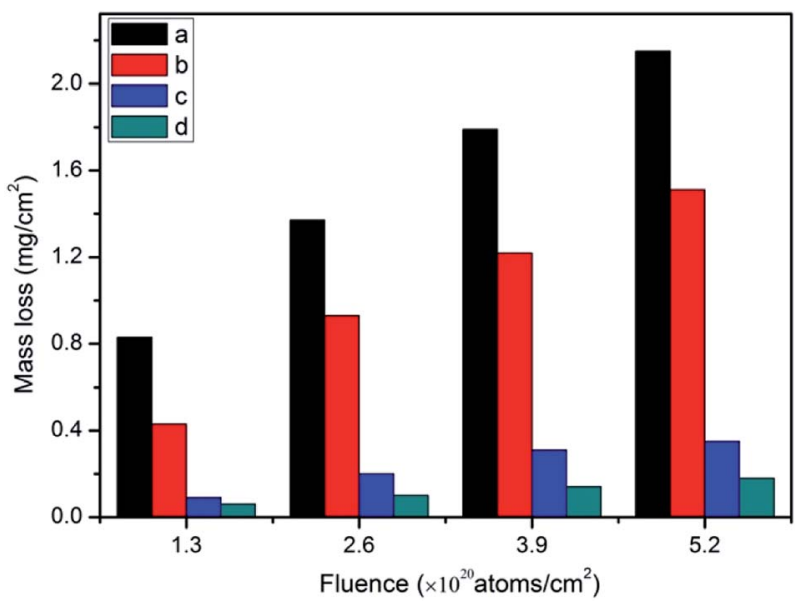

Fig. 7 Mass loss of the samples: (a) R-Ag/PI; (b) Ag/PI; (c) $10 w t \%$ Foc/ $\mathrm{Ag} / \mathrm{Pl}$; and (d) $20 \mathrm{wt} \% \mathrm{Foc} / \mathrm{Ag} / \mathrm{PI}$

\subsection{AO resistance}

3.4.1 Mass loss. Mass loss data: (a) R-Ag/PI; (b) Ag/PI; (c) $10 \mathrm{wt} \% \mathrm{Foc} / \mathrm{Ag} / \mathrm{PI}$; (d) $20 \mathrm{wt} \% \mathrm{Foc} / \mathrm{Ag} / \mathrm{PI}$ at different fluences are listed in Fig. 7. The average mass losses of $\mathrm{R}-\mathrm{Ag} / \mathrm{PI}$ at each $\mathrm{AO}$ fluence of $1.3 \times 10^{20}, 2.6 \times 10^{20}, 3.9 \times 10^{20}$, and $5.2 \times 10^{20}$ atoms per $\mathrm{cm}^{2}$ were $0.83,1.37,1.79$, and $2.15 \mathrm{mg} \mathrm{cm}^{-2}$, respectively. The mass losses of $\mathrm{R}-\mathrm{Ag} / \mathrm{PI}$ increased markedly as the AO fluence increased. Ag/PI showed an identical trend. However, the mass loss of $\mathrm{Ag} / \mathrm{PI}$ was slightly decreased compared with R-Ag/PI, which might be because of the wellestablished silver layers after reimidization resulting in a loose porous silver oxide layer that fell off. The mass loss of Ag/ $\mathrm{PI}$ at an AO fluence of $5.2 \times 10^{20}$ atoms per $\mathrm{cm}^{2}$ was $1.51 \mathrm{mg}$ $\mathrm{cm}^{-2}$.

The mass losses of $10 \mathrm{wt} \% \mathrm{Foc} / \mathrm{Ag} / \mathrm{PI}$ and $20 \mathrm{wt} \% \mathrm{Foc} / \mathrm{Ag} / \mathrm{PI}$ were markedly reduced compared with $\mathrm{R}-\mathrm{Ag} / \mathrm{PI}$ and $\mathrm{Ag} / \mathrm{PI}$. This was the result of modification by PAAS. They showed the same trend as $\mathrm{R}-\mathrm{Ag} / \mathrm{PI}$ as $\mathrm{AO}$ fluence increased. The average mass losses of $10 \mathrm{wt} \% \mathrm{Foc} / \mathrm{Ag} / \mathrm{PI}$ and $20 \mathrm{wt} \% \mathrm{Foc} / \mathrm{Ag} / \mathrm{PI}$ at an AO fluence of $5.2 \times 10^{20}$ atoms per $\mathrm{cm}^{2}$ were 0.35 and $0.18 \mathrm{mg} \mathrm{cm}^{-2}$ $20 \mathrm{wt} \% \mathrm{Foc} / \mathrm{Ag} / \mathrm{PI}$ had a lower mass loss than $10 \mathrm{wt} \% \mathrm{Foc} / \mathrm{Ag} / \mathrm{PI}$, because $10 \mathrm{wt} \%$ PAAS cannot fully cover the silver layers on the surface of self-metalized films. This can be seen in the SEM and XPS data.

3.4.2 Surface morphology change. SEM pictures of the samples are listed in Fig. 8: (a)-(d) R-Ag/PI at fluences of 1.3, 2.6, 3.9, and $5.2 \times 10^{20}$ atoms per $\mathrm{cm}^{2}$, respectively; (e)-(h) Ag/PI at fluences of $1.3,2.6,3.9$, and $5.2 \times 10^{20}$ atoms per $\mathrm{cm}^{2}$,
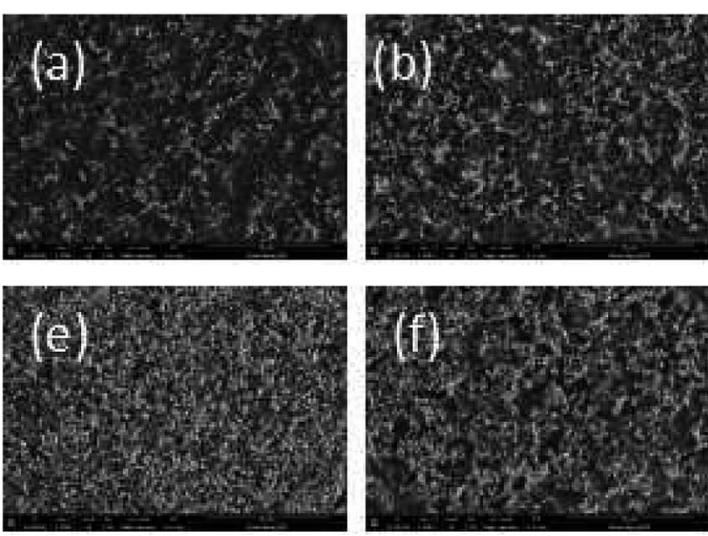
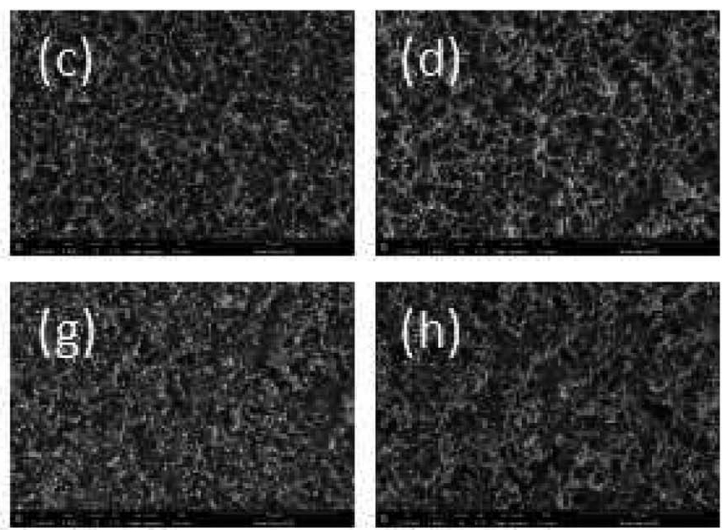

Fig. 8 SEM pictures of the samples: (a)-(d) R-Ag/PI at fluences of 1.3, 2.6, 3.9 and $5.2 \times 10^{20}$ atoms per $\mathrm{cm}^{2}$, respectively; (e)-(h) Ag/PI at fluences of $1.3,2.6,3.9$ and $5.2 \times 10^{20}$ atoms per $\mathrm{cm}^{2}$, respectively.
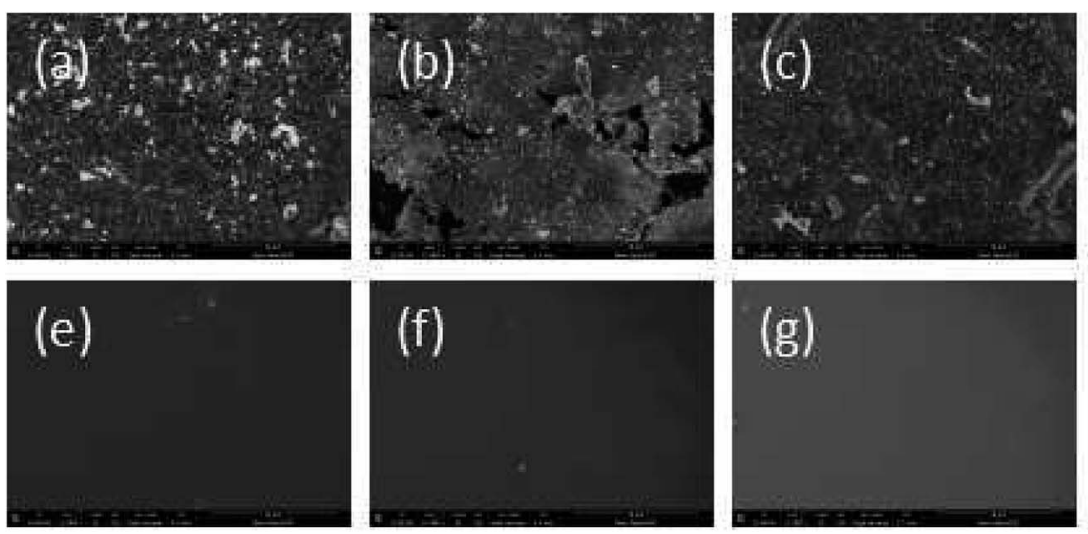

Fig. 9 SEM pictures of the samples: (a)-(d) $10 \mathrm{wt} \%$ Foc/Ag/PI; (e)-(h) $20 \mathrm{wt} \%$ Foc/Ag/PI at fluences of 1.3, 2.6, 3.9, and 5.2 $\times 10^{20}$ atoms per $\mathrm{cm}^{2}$, respectively. 
Table 2 Surface atomic concentration (in percent) determined from XPS survey scans before and after AO exposure to a fluence of $5.2 \times$ $10^{20}$ atoms per $\mathrm{cm}^{2}$

\begin{tabular}{|c|c|c|c|c|c|c|}
\hline \multirow[b]{2}{*}{ Materials } & & \multicolumn{5}{|l|}{ At $\%$} \\
\hline & & $\mathrm{C} 1 \mathrm{~s}$ & N1s & O1s & Ag3d & Si2p \\
\hline \multirow[t]{2}{*}{$\mathrm{R}-\mathrm{Ag} / \mathrm{PI}$} & Before AO & 51.04 & 9.11 & 14.23 & 25.63 & - \\
\hline & After AO & 59.94 & 5.05 & 21.81 & 12.66 & - \\
\hline \multirow[t]{2}{*}{$\mathrm{Ag} / \mathrm{PI}$} & Before AO & 53.67 & 6.48 & 19.39 & 20.46 & - \\
\hline & After AO & 57.85 & 6.75 & 23.80 & 11.11 & - \\
\hline $10 \mathrm{wt} \%$ & Before AO & 53.60 & 3.16 & 22.48 & 2.08 & 18.73 \\
\hline Foc/Ag/PI & After AO & 35.50 & 7.11 & 29.31 & 13.61 & 12.53 \\
\hline $20 \mathrm{wt} \%$ & Before AO & 55.24 & 3.36 & 22.33 & - & 19.07 \\
\hline Foc/Ag/PI & After AO & 41.88 & 17.34 & 25.22 & 0.35 & 9.43 \\
\hline
\end{tabular}

respectively. SEM showed fewer silver particles on the surfaces of R-Ag/PI and Ag/PI after exposure to AO. The surfaces attained a "carpet condition" that was more obvious as the AO fluence increased. This might be because silver particles were oxidized by AO to form silver oxide that became loose and fell off.

The surface morphology change was also measured after surface modification by PAAS. SEM data of the samples are shown in Fig. 9: (a)-(d) $10 \mathrm{wt} \% \mathrm{Foc} / \mathrm{Ag} / \mathrm{PI}$ at fluences of 1.3, 2.6, 3.9 , and $5.2 \times 10^{20}$ atoms per $\mathrm{cm}^{2}$, respectively; (e)-(h) $20 \mathrm{wt} \%$ Foc/Ag/PI at fluences of 1.3, 2.6, 3.9, and $5.2 \times 10^{20}$ atoms per $\mathrm{cm}^{2}$, respectively. After exposure to AO, the surface morphology on $10 \mathrm{wt} \% \mathrm{Foc} / \mathrm{Ag} / \mathrm{PI}$ was distinctly improved compared to Fig. 8. However, the surface also had some conspicuous drawbacks that will affect the properties of the self-metalized films. $20 \mathrm{wt} \% \mathrm{Foc} / \mathrm{Ag} / \mathrm{PI}$ had a smooth surface at fluences of 1.3, 2.6, 3.9 , and $5.2 \times 10^{20}$ atoms per $\mathrm{cm}^{2}$. This might be attributed to full coverage over the self-metalized films by PAAS, and a compact protective layer coming into being after exposure to AO. The protective layer structure is confirmed in Section 3.4.3.

3.4.3 Surface compositional and structural change. Surface atomic concentrations (in percent) determined from XPS survey scans before and after AO exposure to a fluence of $5.2 \times 10^{20}$ atoms per $\mathrm{cm}^{2}$ are summarized in Table 2. For $\mathrm{R}-\mathrm{Ag} / \mathrm{PI}$ and $\mathrm{Ag} /$ $\mathrm{PI}$, the concentration of the irradiated-surface silver atoms dramatically decreased to almost half compared to that of the

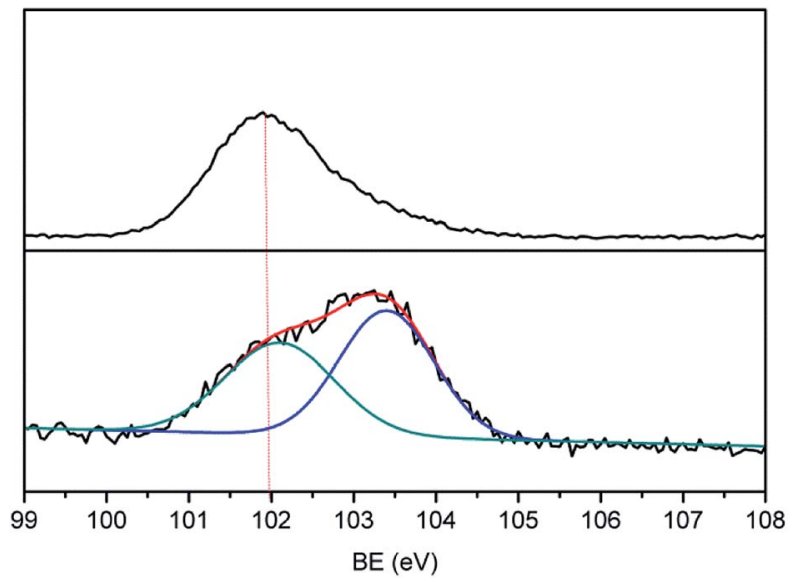

Fig. 10 High-resolution Si2p spectra of 10 wt\% Foc/Ag/PI.

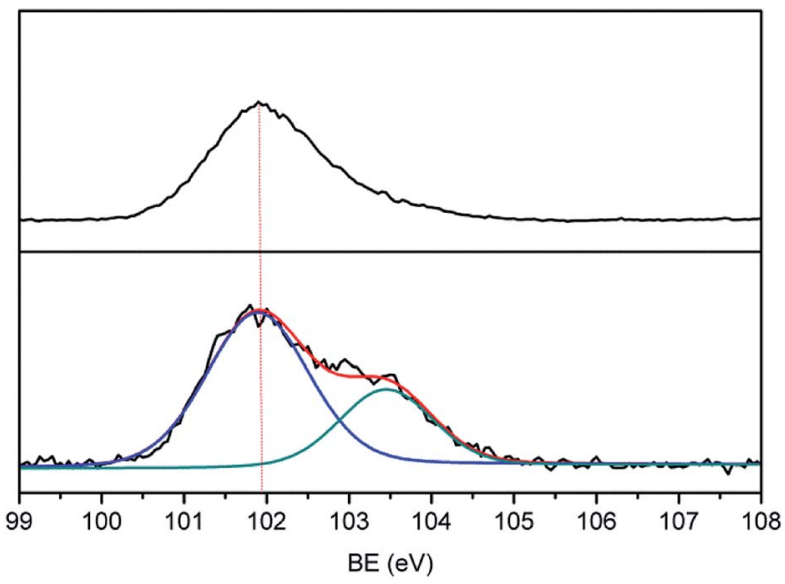

Fig. 11 High-resolution Si2p spectra of 20 wt\% Foc/Ag/PI.

pristine samples. This phenomenon was due to the reaction of silver on the surface with AO to form a loosened silver oxide that became detached. The concentration of the irradiated-surface silver atoms on $10 \mathrm{wt} \% \mathrm{Foc} / \mathrm{Ag} / \mathrm{PI}$ increased from $2.08 \%$ to $13.61 \%$, and this might be because the uncovered silver oxide did not detach because of the existence of a sparse protective layer derived from $10 \mathrm{wt} \% \mathrm{Foc} / \mathrm{Ag} / \mathrm{PI}$. The concentration of the irradiated-surface silver atoms on $20 \mathrm{wt} \% \mathrm{Foc} / \mathrm{Ag} / \mathrm{PI}$ was only $0.35 \%$.

A protective layer structure was confirmed by high-resolution XPS spectra (see Fig. 10 and 11). The Si2p peak position for the pristine surfaces was located at $c a$. $102.0 \mathrm{eV}$, belonging to the $\mathrm{O}-$ $\mathrm{Si}-\mathrm{O}$ in the siloxane. However, the Si2p peak after AO exposure to a fluence of $5.2 \times 10^{20}$ atoms per $\mathrm{cm}^{2}$ was divided into two different shifts located at ca. 102.0 and $103.6 \mathrm{eV}$. The existence of the binding energy of $c a .102 .0 \mathrm{eV}$ showed that the $\mathrm{O}-\mathrm{Si}-\mathrm{O}$ bonds derived from siloxane did not disappear. However, the presence of a new binding energy at $c a$. $103.6 \mathrm{eV}$ indicated the formation of a surface-SiO ${ }_{2}$-type layer. Finally, the passivated $\mathrm{SiO}_{2}$ layer can prevent surface erosion by AO. Thus, $20 \mathrm{wt} \% \mathrm{Foc} /$ $\mathrm{Ag} / \mathrm{PI}$ had a lower mass loss and smoother surface than the others. The erosion and protective mechanism of silvered polyimide films and surface modification by PAAS are depicted in Fig. 12.

(a)
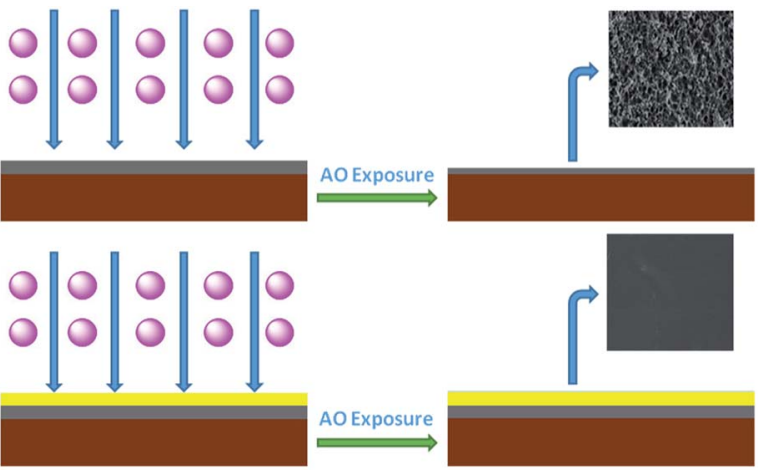

Fig. 12 Schematic of the erosion or protective mechanism: (a) silvered polyimide films and (b) surface modification by PAAS. 


\section{Conclusions}

We evaluated the tolerance of silvered polyimide films synthesized by an in situ self-metalization method against AO. Mass losses of R-Ag/PI were markedly increased as the AO fluence increased, and $\mathrm{Ag} / \mathrm{PI}$ showed an identical trend. Compared to SEM pictures before exposure to $\mathrm{AO}$, silver particles on $\mathrm{R}-\mathrm{Ag} / \mathrm{PI}$ and Ag/PI disappeared, and the surfaces attained a "carpet condition" that was more obvious as the AO fluence increased.

After surface modification by poly(siloxane amic acid) ammonium salts, the AO resistance and the impacts on mass loss, surface morphology, and surface compositions were also evaluated. $20 \mathrm{wt} \% \mathrm{Foc} / \mathrm{Ag} / \mathrm{PI}$ had the lowest mass loss and the smoothest surface. This was due to the formation of a compact surface-SiO $\mathrm{S}_{2}$-type layer.

\section{Conflicts of interest}

There are no conflicts to declare.

\section{Acknowledgements}

Financial support from the foundation (JSHS2015203B002) is gratefully acknowledged.

\section{References}

1 D. J. Liaw, K. L. Wang, Y. C. Huang, K. R. Lee, J. Y. Lai and C. S. Ha, Prog. Polym. Sci., 2012, 37, 907-974.

2 X. Ma, R. Swaidan, Y. Belmabkhout, Y. Zhu, E. Litwiller, M. Jouiad, I. Pinnau and Y. Han, Macromolecules, 2012, 45, 3841-3849.

3 K. L. Mittal, Polyimides and Other High Temperature Polymers: synthesis, characterization, and applications, Brill, vol. 4, 2007.

4 M. Ghosh, Polyimides: Fundamentals and Applications, Belgeler Com, 1996, vol. 2004, ch. xii, p. 404.

5 M. Ding, Prog. Polym. Sci., 2007, 32, 623-668.
6 J. E. Prussing, J. Guid. Control Dyn., 2004, 23, 768.

7 R. E. Freeland, G. D. Bilyeu and G. R. Veal, Acta Astronaut., 1996, 38, 251-260.

8 Z. Wu, D. Wu, S. Qi, Z. Teng and R. Jin, Thin Solid Films, 2005, 493, 179-184.

9 S. Qi, Z. Wu, D. Wu, A. Wencai Wang and R. Jin, Chem. Mater., 2007, 19, 393-401.

10 A. C. Tribble, The Space Environment: Implications for Spacecraft Design, Princeton Univ Pr, 2003.

11 H. Shimamura and T. Nakamura, Polym. Degrad. Stab., 2009, 94, 1389-1396.

12 N. Atar, E. Grossman, I. Gouzman, A. Bolker, V. J. Murray, B. C. Marshall, M. Qian, T. K. Minton and Y. Hanein, ACS Appl. Mater. Interfaces, 2015, 7, 12047-12056.

13 T. K. Minton, M. E. Wright, S. J. Tomczak, S. A. Marquez, L. Shen, A. L. Brunsvold, R. Cooper, J. Zhang, V. Vij and A. J. Guenthner, ACS Appl. Mater. Interfaces, 2012, 4, 492-502. 14 M. R. Reddy, J. Mater. Sci., 1995, 30, 281-307.

15 J. W. Connell, J. Smith, G. Joseph, C. G. Kalil and E. J. Siochi, Polym. Adv. Technol., 2015, 9, 11-19.

16 S. Packirisamy, D. Schwam and M. H. Litt, J. Mater. Sci., 1995, 30, 308-320.

17 L. Li, J. C. Yang and T. K. Minton, J. Phys. Chem. C, 2007, 111, 6763-6771.

18 M. K. Bhan, P. K. Nag, G. P. Miller and J. C. Gregory, J. Vac. Sci. Technol., A, 1994, 12, 699-706.

19 L. Hu, M. Li, C. Xu and Y. Luo, Thin Solid Films, 2011, 520, 1063-1068.

20 E. Miyazaki, M. Tagawa, K. Yokota, R. Yokota, Y. Kimoto and J. Ishizawa, Acta Astronaut., 2010, 66, 922-928.

21 D. Cai, J. Su, M. Huang, Y. Liu, J. Wang and L. Dai, Polym. Degrad. Stab., 2011, 96, 2174-2180.

22 S. CV, A. Cavaleiro and S. Carvalho, Appl. Surf. Sci., 2015, 346, 240-247.

23 Z. Cao, C. Liu, C. Qu, X. Zhao, D. Wang, C. Chen, X. Hou, L. Li and G. Zhu, Eur. Polym. J., 2017, 96, 393-402. 